\title{
Molecular rotational diffusion detected by differential fluorescence energy
}

\author{
Jorge R. Torga ${ }^{\mathrm{a}, *}$, Mario C. Marconi ${ }^{\mathrm{a}}$, R. Martín Negri ${ }^{\mathrm{b}}$, Pedro F. Aramendía ${ }^{\mathrm{b}}$ \\ a Laboratorio de Electrónica Cuántica, Departamento de Física, Pabellon, Facultad de Ciencias Exactas y Naturales, \\ Universidad de Buenos Aires Ciudad Universitaria, Buenos Aires 1428, Argentina \\ b INQUIMAE, Departamento de Química Inorgánica, Analitica y Química, Física, Facultad de Ciencias Exactas y Naturales, \\ Universidad de Buenos Aires Ciudad Universitaria, Buenos Aires 1428, Argentina
}

Received 17 August 1999

\begin{abstract}
In this paper, we present a complete study of the influence of the molecular rotational diffusion movement in the fluorescence signal when a sample is excited by two short pulses. The study includes the calculations of this effect for ellipsoidal molecules. Experimental results are presented that show a fluorescence behavior influenced by the molecular rotational diffusion movement with temporal characteristics that agree with our calculations. (C) 2000 Elsevier Science B.V. All rights reserved.
\end{abstract}

\section{Introduction}

The study of the rotational diffusion movement of molecules in liquid solvents is a powerful method applied to the understanding of the complex interactions in the solute-solvent system. The characteristic time of this phenomenon can be evaluated by different techniques [1-3], both in steady state or in time-resolved experiments which allow us the evaluation of parameters related with the molecular shape and the influence of the solvent in its movement. Interesting data acquired by time resolved fluorescence and absorption, allow us to obtain detailed information of ultrafast relaxation of the solute molecules by directly mea-

\footnotetext{
${ }^{*}$ Corresponding author. Present address: Universidad Technológica Nacional, Regional Delta, San Martin 1171 (2804), Campana, Buenos Aires, Argentina. Fax: +54-1-782-7647.

E-mail address: torga@df.uba.ar (J.R. Torga).
}

suring the depolarization produced after a short excitation period.

Using ultrashort laser pulses, the time resolution can achieve the subpicosecond time scale. Examples of techniques that make use of ultrashort laser excitation are polarization spectroscopy and anisotropic absorption, in which a pump and probe scheme is used to measure time-resolved absorption [4-9]. Great insight in these studies was obtained by fluorescence up-conversion $[10,11]$ where a cross-correlation between the short pulse excitation and the fluorescence is used to measure the last signal with subpicosecond temporal resolution. Reviews and comments on different techniques applied to these measurements can be found in Refs. [2,3,12].

Recently, we proposed a novel technique that allows the measurement of rotational diffusion times based on the study of the fluorescence produced when two linearly polarized laser pulses 
excite the sample. Both pulses are separated in time by a variable delay using a conventional pump and probe set-up. When absorbed, the first pulse induces a non-homogeneous spatial distribution in the population of excited molecules due to the anisotropy introduced by the electric field of the light. The second pulse then interacts with the sample when there are still some molecules in the excited state. Under this condition, the second excitation pulse finds a non-homogeneous spatial distribution of absorbing (ground state) molecules. This anisotropy changes the total fluorescence produced by the double excitation, which is collected as a function of the time delay. The analysis of this signal, allows us to retrieve molecular rotational times. A proposed experiment to measure this characteristic time for the simple case of spherical molecules and a discussion of the influence of the finite pulse width of the excitation was presented in previous works [13-15].

In this paper, we present a detailed analysis of how the rotational diffusion influences the fluorescence when the sample is excited with two short pulses. This study includes the expressions for the integrated fluorescence energy for ellipsoidal molecules. The influence of the double pulse excitation and the photoselection is made evident through a parameter defined in this work as the "differential fluorescence energy", obtained as the change in the fluorescence signal produced by this particular double pulse excitation, compared with twice the fluorescence obtained with the first pulse. Experimental results using a sample of Rhodamine 101 in ethylene glycol show the effect predicted by the calculations.

\section{Initial considerations}

The set-up of the two pulses excitation method is schematically shown in Fig. 1. We consider the fluorescent sample homogeneous before the excitation by the first pulse, i.e. the absorbing dipoles are randomly oriented. The absorbing molecules are considered ellipsoidal and each molecule having an absorbing dipole moment $\boldsymbol{\mu}$ with the same

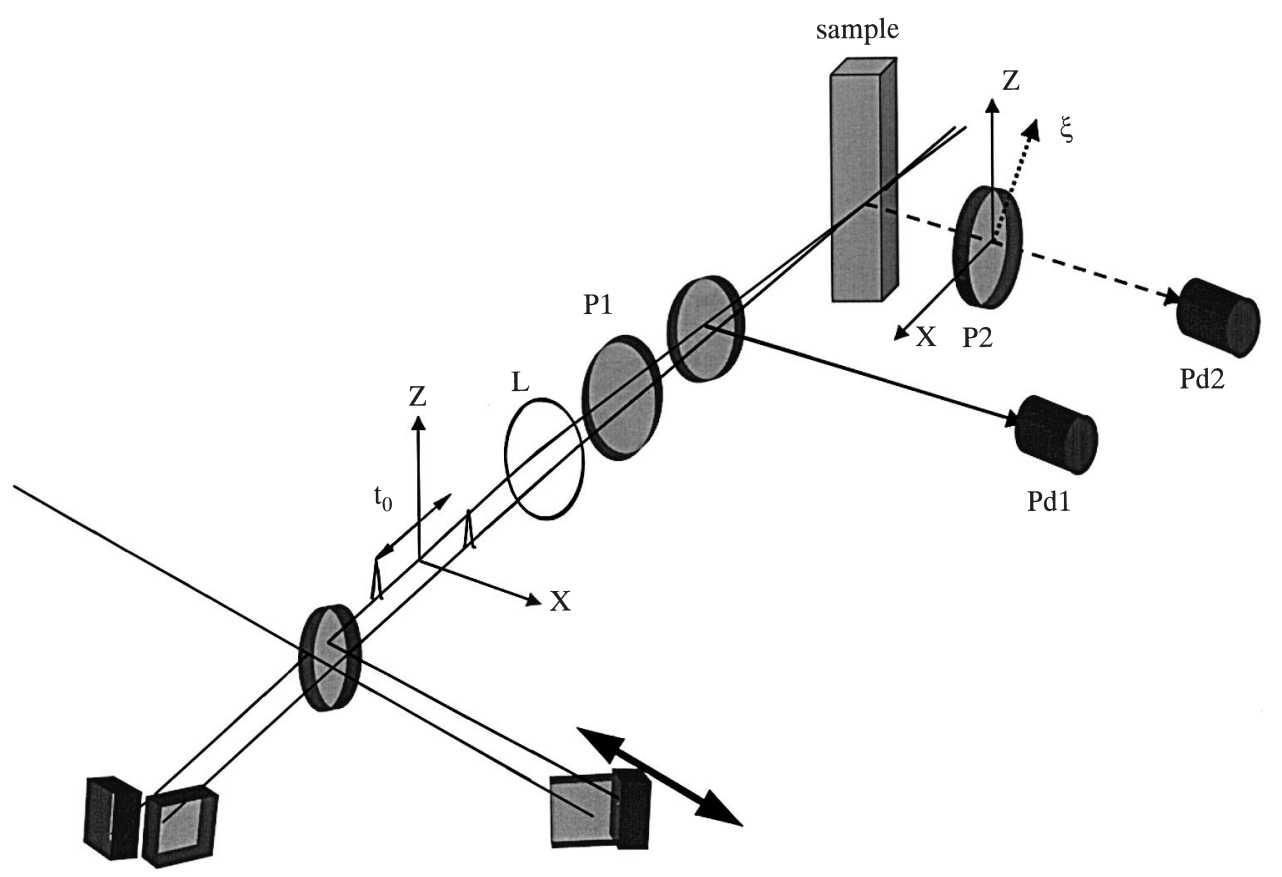

Fig. 1. Double pulse excitation set-up. Pd1 and Pd2: large area silicon photodiodes used to measure the excitation pulse energy and the fluorescence energy, respectively. P1 and P2 are polarizers. L is the focusing lens. 
orientation as the emission dipole moment. We also consider that each molecule is a two level system and that the de-excitation is exclusively by radiative decay. This is a good approximation for Rhodamine 101 with the fluorescence quantum efficiency $\phi_{\mathrm{f}}=1$ [16]. In general, other deactivation channels leading to the ground state by nonradiative pathways only shorten the excited state lifetime but do not influence rotational reorientation as both transition dipoles are parallel, and thus do not alter the conclusions and analysis that follows.

The excitation pulse is assumed linearly polarized, and having a temporal dependence corresponding to a delta function with a total energy $a$.

We define $n_{\mathrm{e}}(\Omega, t)$ the density of excited molecules and $n_{\mathrm{g}}(\Omega, t)$ the density of molecules in the ground state as a function of the dipole orientation defined by the angular coordinates $\Omega$, and the time $t$. If $n_{0}$ is the total density of fluorophores in the solution before the excitation, then:

$n_{\mathrm{g}}(\Omega, t)+n_{\mathrm{e}}(\Omega, t)=n_{0} \quad \forall t \geqslant 0$.

This condition assumes that excited and ground state molecules have the same rotational diffusion constant. The evolution equation for $n_{\mathrm{e}}(\Omega, t)$ is $[13,17]$ :

$$
\begin{aligned}
\frac{\partial}{\partial t} n_{\mathrm{e}}(\Omega, t)= & K^{\prime}(\boldsymbol{\mu} \cdot \mathbf{Z})^{2} n_{\mathrm{g}}(\Omega, t)-\frac{n_{\mathrm{e}}(\Omega, t)}{\tau} \\
& +H n_{\mathrm{e}}(\Omega, t) .
\end{aligned}
$$

Eq. (2) has three terms corresponding to the excitation, the fluorescence decay, and the diffusion term.

In the second term, $\tau$ represents the fluorescence lifetime. In the excitation term, the factor $K^{\prime}$ represents the fraction of molecules excited in the sample per unit of time and is defined as

$K^{\prime}(t)=\sigma \rho \frac{I(t)}{h v s}$,

where $\sigma$ is the absorption cross-section, $\rho$ is the quantum efficiency, $h v$ is the excitation photon energy, $s$ is the spot area and $I(t)$ is the pulse intensity. The third term, corresponding to the ro- tational diffusion of excited molecules, has the diffusion operator $H$, defined as

$H=\sum_{i, j} \mathbf{L}_{i} D_{i j} \mathbf{L}_{j}$

where $D_{i j}$ are the components of the diffusion tensor (D) and $\mathbf{L}_{i}$ are the components of the angular momentum of the molecule.

To describe the system, we will use two sets of Cartesian coordinates, $(x, y, z)$ fixed to the molecule and coincident with its three principal axis, and $(X, Y, Z)$ fixed to the laboratory as is schematically shown in Fig. 2. The excitation pulse is polarized along the $Z$ direction and $Y$ is the propagation direction. $\alpha, \beta, \gamma$ are the Euler angles (Fig. 2) as defined by Rose [18].

Just after the excitation by the first pulse, and assuming that the pulse width $(T)$ is shorter than the diffusion time and fluorescent time, Eq. (2) can be solved to evaluate the initial fraction of excited molecules $n_{\mathrm{e}}$. In this operation, we defined

$K=\int_{0}^{\infty} K^{\prime}(t) \mathrm{d} t=\sigma \rho \frac{a}{h v s}$.

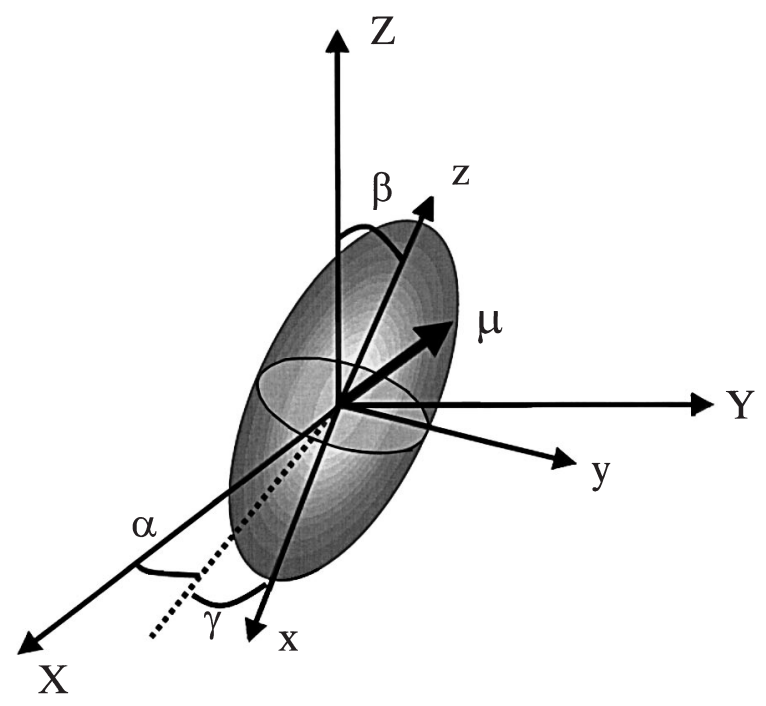

Fig. 2. The two sets of Cartesian coordinates used to describe the system, $(x, y, z)$ fixed to the molecule and coincident with its three principal axis, and $(X, Y, Z)$ fixed to the laboratory. 
Keeping terms up to the second order in $K$, we obtain for the excited state molecules:

$n_{\mathrm{e}}\left(\Omega, t=0^{+}, K\right)=n_{0}\left[K(\boldsymbol{\mu} \cdot \mathbf{Z})^{2}-\frac{1}{2} K^{2}(\boldsymbol{\mu} \cdot \mathbf{Z})^{4}\right]$.

Integration in the angular coordinates gives the initial ratio of excited molecules to the population of the ground state molecules:

$\varepsilon=\frac{n_{\mathrm{e}}\left(t=0^{+}\right)}{n_{0}}=\frac{2 \pi}{15}\left(10 K-3 K^{2}\right)$.

Using typical values of a dye molecule, $\sigma=10^{-16} \mathrm{~cm}^{2}, \rho \cong 1$, and considering an excitation pulse centered at $\lambda=500 \mathrm{~nm}$, focused to an area $s=10^{-4} \mathrm{~cm}^{2}$, with energy $a=1 \mathrm{~nJ}$ and a pulse width $T=100$ fs the evaluation of Eq. (5) yields $K \cong 210^{-3}$. With these values, expression (7) results in a fraction $\varepsilon=0.004$.

\section{Solution of the diffusion term after a double excitation}

Favro has shown that the evolution of the population density of excited molecules corresponding to the diffusion term of Eq. (2) can be written for $t>0$ as [19-21]:

$n_{\mathrm{e}}(\Omega, t, K)=\sum_{l k m} a_{l k m}(K) \Psi_{l k m}(\Omega) \exp \left(-E_{l k m} t\right)$,

where $\Psi_{l k m}(\Omega)$ and $E_{l k m}$ are the eigenfunctions and the eigenvalues of the $H$ operator and $a_{l k m}(K)$ are the coefficients obtained in the decomposition [20].

The second pulse arrives at the sample with a delay time $t_{0}$. We suppose that this pulse is identical to the first one and that the delay time $t_{0}$ is smaller than the fluorescence decay time $\tau$. With these assumptions the total excitation intensity is

$I(t)=a \delta(t)+a \delta\left(t-t_{0}\right)$.

When the second pulse arrives to the sample, there will be still some molecules in the excited state $\left(t_{0}<\tau\right)$. Additionally, the system keeps memory of the photoselection produced by the first pulse if $t_{0}<1 / D$ (where $D=(1 / 3 \operatorname{tr}(\mathbf{D}))$ ) and consequently it will find a non-homogeneous dis- tribution of absorbing (ground state) dipoles. Due to this effect the fluorescence produced by the second pulse will be lower than the fluorescence produced by the first pulse.

We evaluate this effect by the "differential density" $n_{\mathrm{d}}(\Omega, t, K)$, defined as

$$
\begin{gathered}
n_{\mathrm{d}}\left(\Omega, t=t_{0}, K\right)=n_{\mathrm{e}}\left(\Omega, t=t_{0}^{-}, K\right) \\
\times\left[K(\boldsymbol{\mu} \cdot \mathbf{Z})^{2}-\frac{1}{2} K^{2}(\boldsymbol{\mu} \cdot \mathbf{Z})^{4}\right] .
\end{gathered}
$$

The differential density represents the molecules that were not excited by the second pulse because they are still in the excited state after the excitation by the first pulse. $n_{\mathrm{d}}$ depends on the delay between pulses and on the fluorescence and rotational diffusion times. A detailed derivation of the expression (10) can be found in Appendix A.

With expression (10), it is possible to determine the corresponding fluorescence energy $E d_{p}$ (parallel to the $Z$ axis) and $E d_{e}$ (perpendicular to $Z$ axis) integrating $n_{\mathrm{d}}(\Omega, t, K)$ in the solid angle $\Omega$ and in time. The detailed calculation of this expression can be found in Appendix A:

$$
\begin{gathered}
E d_{p}=\frac{1}{\tau} \int_{\Omega} \int_{t_{0}}^{\infty} n_{\mathrm{d}}\left(\alpha, \beta, t, t_{0}\right)(\mu \cdot Z)^{2} \mathrm{~d} \Omega \mathrm{d} t, \\
E d_{e}=\frac{1}{2 \tau} \int_{\Omega} \int_{t_{0}}^{\infty} n_{\mathrm{d}}\left(\alpha, \beta, t, t_{0}\right)\left[(\mu \cdot X)^{2}\right. \\
\left.+(\mu \cdot Y)^{2}\right] \mathrm{d} \Omega \mathrm{d} t
\end{gathered}
$$

After integration a general expression for $E d_{p}$ and $E d_{e}$ can be obtained for the case considered in this paper, dipole parallel to the molecular symmetry axis ( $z$ axis) or in the plane perpendicular to this axis ( $x$ axis). The general form is given by

$$
\begin{aligned}
E d_{i}=\exp & \left(-\frac{t_{0}}{\tau}\right)\left(A_{i}+B_{i} \exp \left(-\frac{t_{0}}{\Phi_{2}}\right)\right. \\
& \left.+C_{i} \exp \left(-\frac{t_{0}}{\Phi_{1}}\right)\right) \text { for } i=p, e,
\end{aligned}
$$

where $\Phi_{1}$ and $\Phi_{2}$ are rotational diffusion times defined as

$\Phi_{1}=\frac{1}{6 D_{\|}}, \quad \Phi_{2}=\frac{1}{2 D_{\|}+4 D_{\perp}}$. 
In the most general case $A, B, C$ are coefficients that depend on $\tau, \Phi_{1}$ and $\Phi_{2}$ and on the orientation of the dipole relative to the molecular axis. A detailed analysis in two particular cases of the dipole parallel to $z$ and parallel to $x$ are discussed in paragraphs 4 and 5 .

The differential energy represents the difference between the total fluorescence produced by first pulse and the total fluorescence energy produced by the second pulse. As is shown in Eq. (13), this magnitude depends on the time delay between pulses and its dependence has two contributions. The first contribution depends on the fluorescence time and is produced by the difference in the population of the ground state that find the two pulses in the excitation. The second one depends on the rotational times and is produced by the difference in the orientational distribution that the first and the second pulse find in the moment of the excitation. In this way, measuring the differential energy as a function of time delay, rotational diffusion can be obtained.

The differential energies can be measured collecting the fluorescence through a polarizer. We assume that the polarizer is located in the $\mathbf{X}-\mathbf{Z}$ plane (Fig. 1). If we define $\xi$ as the polarizer axis in the detection system, $E d_{p}$ is obtained when $\xi \| \mathbf{Z}$ and $E d_{e}$ when $\xi \| \mathbf{X}$.

We can define the total differential fluorescence energy as

$E d_{t}=\frac{1}{3}\left(E d_{p}+2 E d_{e}\right)$.

$E d_{t}$ is obtained when $\xi$ is oriented as the magic angle, defined as the angle in which the anisotropy produced for the linear excitation is cancelled. This corresponds to an angle of $54.7^{\circ}$ between $\xi$ and $\mathbf{X}$. Defined in this way, $E d_{t}$ results in an expression equal to Eq. (13).

Another magnitude that we may define is the non-polarized differential energy as

$E d_{\mathrm{np}}=E d_{p}+E d_{e}$

$E d_{\text {np }}$ is the differential fluorescence energy when the fluorescence is collected without selecting a direction of polarization. $E d_{\mathrm{np}}$ has the general form given in Eq. (13).
In the next two paragraphs, we will consider two particular situations in which the dipole moment is oriented along the symmetry axis of the molecule ( $z$ axis) and orthogonal to this axis ( $x$ axis).

\section{Dipole in the $z$ axis}

The first case considered is when the dipole $\boldsymbol{\mu}$ is parallel to the $z$ axis, in the direction orthogonal to the symmetry plane. To obtain the expression for differential population we use Eq. (A.5) in Appendix A. In this case, only the coefficients $b_{00}, b_{20}$, $b_{40}$ don't give null values. In Appendix B, we give a complete expression for each coefficient.

Replacing expressions (A.7) and (A.8) in Eq. (15), we obtain

$$
E d_{t}=\frac{K^{2} \tau}{270 \pi} \exp \left(-\frac{t_{0}}{\tau}\right)\left(5+4 \exp \left(-\frac{t_{0}}{\Phi_{1}}\right)\right) .
$$

In this case, the differential energy has only one rotation time ( $\Phi_{1}$ as defined in Eq. (14)). Fig. 3 is a plot of $E d_{t}$ as a function of the delay time $t_{0}$ normalized to the fluorescence time $\tau$ for different values of the ratio $\Phi_{1} / \tau$. Expression (17) has three parameters $\left(K, \Phi_{1}\right.$ and $\left.\tau\right)$. The ratio $\Phi_{1} / \tau$ has a great spread of values, and it depends primarily on the "friction" in the solute-solvent interaction. To

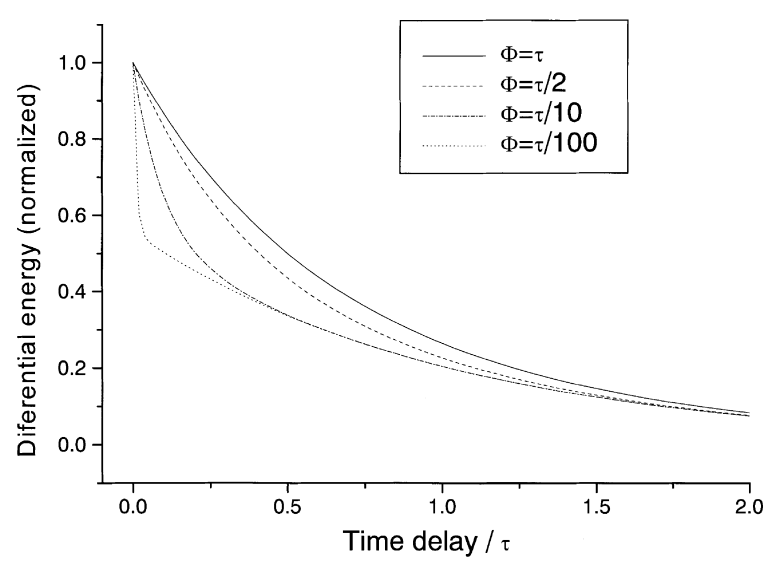

Fig. 3. Differential energy as a function of the normalized time delay $t_{0} / \tau$ for the case in which the dipole is along the $z$ axis for different values of $\Phi_{1}$. 
plot the curves in Fig. 3, we choose as a reference $K=10^{-3}$ and $\tau / 100 \leqslant \Phi_{1} \leqslant \tau$, considering that this is the typical range of variation for the ratio $\Phi_{1} / \tau$.

In all cases, the curves show a bi-exponential behaviour with characteristic times given by $\left(\Phi_{1}^{-1}+\tau^{-1}\right)^{-1}$ and $\tau$.

In the case that the fluorescence time is much longer than the rotation time, the term $\left(-t_{0} / \tau\right)$ can be considered as a constant if $\Phi_{1} \leqslant t_{0} \ll \tau$. In this situation, $E d_{t}$ depends primarily on the rotational time. When $t_{0}$ increases, the fluorescence exponential decay is noticeable. This behaviour is shown in Fig. 3 when $\Phi_{1}=\tau / 100$. In the opposite situation, when $\Phi_{1} \approx \tau$ the two exponential decays are similar.

\section{Dipole in the $x$ axis}

The second case considered is when the dipole $\boldsymbol{\mu}$ is included in the plane orthogonal to the $z$ axis.

Following the same steps presented in the previous paragraph, we obtain the expressions for the differential population. The $b_{l m}$ no null coefficients are $b_{00}, b_{20}, b_{2 \pm 2}, b_{40}, b_{4 \pm 2}, b_{4 \pm 4}$. The complete expressions are given in Appendix C.

The expression for the total differential energy in this case is

$$
\begin{aligned}
E d_{t}= & \frac{K^{2} \tau}{180 \pi} \exp \left(-\frac{t_{0}}{\tau}\right) \\
& \times\left(5+3 \exp \left(-\frac{t_{0}}{\Phi_{2}}\right)+\exp \left(-\frac{t_{0}}{\Phi_{1}}\right)\right)
\end{aligned}
$$

Here the differential energy has two characteristic rotation times $\Phi_{1}$ and $\Phi_{2}$, that were defined in Eq. (14).

Fig. 4 shows the values of the differential energy as a function of the time delay for different values of rotation times. We impose, as in the previous case, the condition $\tau / 100 \leqslant \Phi_{1}, \Phi_{2} \leqslant 2 \tau$ to select the range of variation for $\Phi_{1}$ and $\Phi_{2}$. In this situation, three exponential times appear as a function of time delay, and the shape of the curves depends on the characteristic relationship between $\Phi_{1}, \Phi_{2}$ and $\tau$.

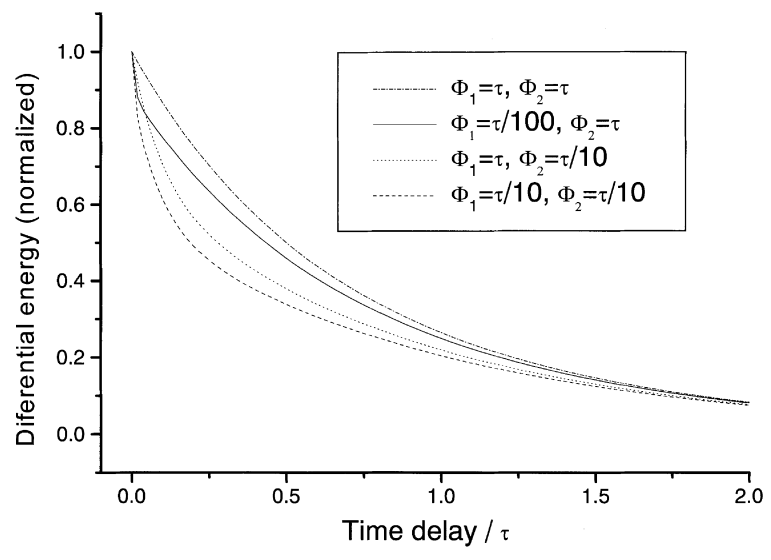

Fig. 4. Differential energy as a function of the normalized time delay $t_{0} / \tau$ for the case in which the dipole is along the $x$ axis for different values of $\Phi_{1}$ and $\Phi_{2}$.

\section{Experiment}

To test the proposed method, we mounted the double pulse excitation scheme shown in Fig. 1. The excitation system was based on a pulsed dye laser producing $800 \mathrm{ps}$ pulses at a repetition rate of $10 \mathrm{~Hz}$. The average energy per pulse was $10 \mathrm{~nJ}$. The excitation pulse was launched in a double pulse excitation set-up capable of introducing a delay time up to $6 \mathrm{~ns}$. Both pulses were polarized in a direction parallel to $Z$ axis and were focused using a $5 \mathrm{~cm}$ focal length lens. The excited volume depends on the focal length of the focusing system and also on the characteristics of the incident beam. Due to this influence, special precautions were taken in collimating the excitation beam. A change in the spot size at the focusing lens, due to a poor collimation of the variable length branch, may cause an unwanted variation in the excited volume, consequently changing the fluorescence energy and masking the effect of the differential energy due to the double pulse excitation. By carefully adjusting the collimation of the laser beam, this influence was minimized. In spite of this careful adjustment, a small variation in the fluorescence signal was observed when the sample was illuminated only with the variable length branch beam when the sliding mirror was displaced. To compensate this small contribution, the fluorescence signal with the double pulse excitation was normalized to the sig- 
nal obtained, when the sample was illuminated only with the variable length branch.

The fluorescence was collected with an optical system with a numerical aperture of 0.5 . The excitation parameters in the experiment yields: $K=0.008$. The total fluorescence was detected using a large area silicon photodiode ( $\mathrm{Pd} 2)$.

Simultaneously, a sample of the excitation pulse was derived before impinging the sample to record the energy for every excitation pulse for normalizing purposes using an identical detector (Pd1). For each delay, a set of 1000 pulses was registered using a sample and hold acquisition circuit followed by two equalized amplifiers and an analog/ digital data converter. From these data, the normalized fluorescence signal was obtained for each delay as the mean value of the 1000 pulses.

The sample used in this test was a solution of Rhodamine 101 in ethylene glycol due to the expected long rotational diffusion time, compatible with the 800 ps pulse that we have in the excitation laser. We assume that this molecule exhibits a behaviour corresponding to only one rotation time $\left(\Phi_{1}\right)$. We measured the steady-state fluorescence anisotropy $\langle r\rangle$ as a function of temperature between $0^{\circ} \mathrm{C}$ and $70^{\circ} \mathrm{C}$. To derive $\Phi_{1}$ from these measurement we assume the relation [1]:

$\langle r\rangle=\frac{r_{0}}{1+\frac{\tau}{\Phi_{1}}}$

with $\Phi_{1} \propto 1 / D_{\perp}$ and $\tau=3.8 \mathrm{~ns}$. After fitting the data, we obtain the rotational diffusion time $\Phi_{1}=3.5 \mathrm{~ns}$ at $294 \mathrm{~K}$, assuming $r_{0}=0.34$.

The same sample was measured with the differential energy technique. The experimental results obtained in this test are shown in Fig. 5. In this case, the plot correspond to the $E d_{p}$.

Changing the delay between pulses, the differential fluorescence energy shows an exponential decay with a time constant in good agreement with the former calculations. The data points in Fig. 5 correspond to the mean value obtained from the 1000 pulses acquired for each delay, and the error bars are the statistical spread of these data points. The solid line is the best exponential fit with a fitting function:

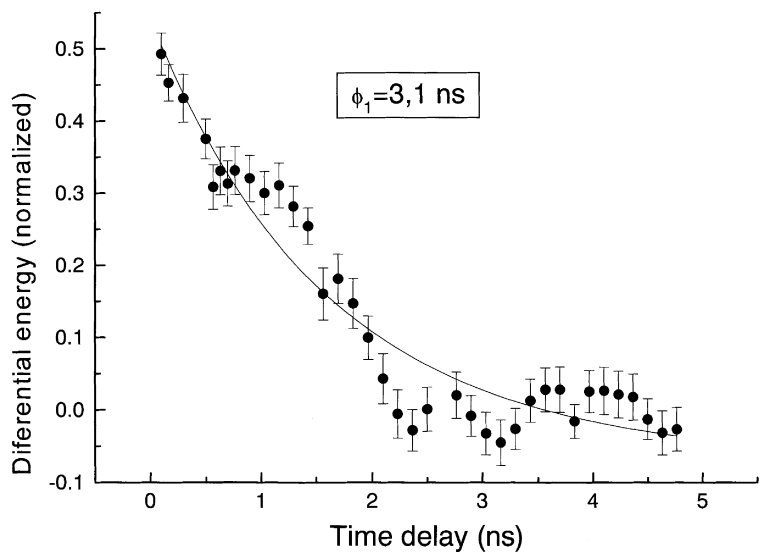

Fig. 5. Measured differential energy as a function of the normalized time delay $t_{0} / \tau$. Each data point is the mean value of 1000 pulses. The solid line is the best fit using the theoretical expression for the differential energy.

$E d_{p}=\exp \left(-\frac{t}{\tau}\right)\left(A+B \exp \left(-\frac{t}{\Phi_{1}}\right)\right)$

This fit yields $\Phi_{1}=3.1$ ns, considering $\tau=3.8 \mathrm{~ns}$.

\section{Conclusions}

In this paper, we present a detailed analysis and preliminary experimental results that make evident the influence of the molecular rotational diffusion movement in the total fluorescence produced when a homogeneous ensemble of fluorophores is excited by two polarized and delayed short pulses. The calculations indicate that the total fluorescence obtained in this case has a dependence on the rotational diffusion time and on the delay between the excitation pulses. To evaluate this dependence, we introduce a magnitude that can be derived from the fluorescence signal: the differential fluorescence energy. Expressions (17) and (18) show that the differential energy presents an exponential dependence as a function of the time delay between the pulses with characteristic time constants given by fluorescence and rotational diffusion times. We found such an exponential decay with values that are in good agreement 
with those obtained from steady-state fluorescence anisotropy measurement. The use of the differential energy presents some advantages: the rotational diffusion times can be obtained in a direct way since only one magnitude (total differential energy) is needed to fit the exponential decay observed as a function of the delay between pulses. In all cases, the curves for $E d_{t}, E d_{p}, E d_{e}$ and $E d_{\text {np }}$ as a function of time delay present a similar behaviour.

As in other correlation techniques, no fast detection is required since the fluorescence energy is integrated in time to obtain the differential energy and the temporal resolution is only limited by the excitation pulse width.

\section{Acknowledgements}

This research was supported by the Universidad de Buenos Aires (grants TX99, TX10) and CONICET (PIP 0639, 0388).

\section{Appendix A}

After the excitation with the second pulse the density of molecules in the excited state can be calculated as

$$
\begin{aligned}
n_{\mathrm{e}}\left(\Omega, t_{0}^{+}\right)= & {\left[n_{0}-n_{\mathrm{e}}\left(\Omega, t_{0}^{-}\right)\right]\left[K(\boldsymbol{\mu} \cdot \mathbf{Z})^{2}-\frac{1}{2} K^{2}(\boldsymbol{\mu} \cdot \mathbf{Z})^{4}\right] } \\
& +n_{\mathrm{e}}\left(\Omega, t_{0}^{-}\right) .
\end{aligned}
$$

In this expression, the first term represents the excited molecules produced only by the second pulse and the second term corresponds to the molecules excited by the first pulse that still remain in the excited state when the second pulse arrives.

Expression (A.1) can be written as

$$
\begin{aligned}
n_{\mathrm{e}}\left(\Omega, t_{0}^{+}\right)= & n_{0}\left[K(\boldsymbol{\mu} \cdot \mathbf{Z})^{2}-\frac{1}{2} K^{2}(\boldsymbol{\mu} \cdot \mathbf{Z})^{4}\right]+n_{\mathrm{e}}\left(\boldsymbol{\Omega}, t_{0}^{-}\right) \\
& -n_{\mathrm{e}}\left(\Omega, t_{0}^{-}\right)\left[K(\boldsymbol{\mu} \cdot \mathbf{Z})^{2}-\frac{1}{2} K^{2}(\boldsymbol{\mu} \cdot \mathbf{Z})^{4}\right] .
\end{aligned}
$$

We define $n_{\mathrm{d}}(\Omega, t, K)$, "the differential density" of excited molecules as

$$
\begin{gathered}
n_{\mathrm{d}}\left(\Omega, t=t_{0}, K\right)=n_{\mathrm{e}}\left(\Omega, t=t_{0}^{-}, K\right) \\
\times\left[K(\boldsymbol{\mu} \cdot \mathbf{Z})^{2}-\frac{1}{2} K^{2}(\boldsymbol{\mu} \cdot \mathbf{Z})^{4}\right] .
\end{gathered}
$$

Considering only quadratic terms in $K$, we can write Eq. (A.3) as

$n_{\mathrm{d}}\left(\Omega, t=t_{0}, K\right)=n_{\mathrm{e}}\left(\Omega, t=t_{0}^{-}, K\right)\left[K(\boldsymbol{\mu} \cdot \mathbf{Z})^{2}\right]$.

With the same formalism presented to obtain Eq. (8), we may obtain an expression for $n_{\mathrm{d}}\left(\Omega, t, t_{0}\right)$ when $t>t_{0}$.

$$
\begin{aligned}
n_{\mathrm{d}}\left(\alpha, \beta, t, t_{0}\right)= & \sum_{l=0}^{2} \sum_{m=-1}^{1} b_{l m}\left(K, t_{0}\right) Y_{l m}(\alpha, \beta) \\
& \times \exp \left(-E_{l m} t\right) .
\end{aligned}
$$

In the particular case of ellipsoidal molecules $\Psi_{l k m}(\Omega)=Y_{l k m}(\alpha, \beta)$, the spherical harmonics and the eigenvalues $E_{l m}$, are defined as:

$E_{l m}=l(l+1) D_{\|}+\left(D_{\perp}-D_{\|}\right) m^{2}$.

In the last expression, the coefficients $D_{\perp}=D_{1}=D_{2}$ and $D_{\|}=D_{3}$ represent the components of the diffusion tensor orthogonal and parallel to the $z$ axis defined in Fig. 2. The $b_{l k m}\left(K, t_{0}\right)$ are obtained in the same way that the $a_{l k m}(K)$ coefficients in Eq. (8).

From Eq. (A.5), we can obtain the expressions for the fluorescence intensity and the fluorescence energy. We calculate the fluorescence energy corresponding to the differential density, $E d_{p}$ (parallel to $Z$ axis) and $E d_{e}$ (perpendicular to $Z$ axis) integrating in the solid angle $\Omega$ and in time:

$$
\begin{aligned}
E d_{p}= & \frac{1}{\tau} \int_{\Omega} \int_{t_{0}}^{\infty} n_{\mathrm{d}}\left(\alpha, \beta, t, t_{0}\right)(\mu \cdot Z)^{2} \mathrm{~d} \Omega \mathrm{d} t \\
E d_{e}= & \frac{1}{2 \tau} \int_{\Omega} \int_{t_{0}}^{\infty} n_{\mathrm{d}}\left(\alpha, \beta, t, t_{0}\right) \\
& \times\left[(\mu \cdot X)^{2}+(\mu \cdot Y)^{2}\right] \mathrm{d} \Omega \mathrm{d} t .
\end{aligned}
$$

\section{Appendix B}

Coefficients $b_{l m}$ used in Eq. (A.5) when the dipole $\boldsymbol{\mu}$ is parallel to the $z$ axis: 


$$
\begin{aligned}
& b_{00}=\frac{K^{2}}{270 \pi^{3 / 2}} \exp \left(-\frac{t_{0}}{\tau}\right)\left(5+6 \exp \left(-\frac{t_{0}}{\Phi_{1}}\right)\right), \\
& b_{20}=\frac{K^{2} \sqrt{5}}{1260 \pi^{3 / 2}} \exp \left(-\frac{t_{0}}{\tau}\right)\left(7+11 \exp \left(-\frac{t_{0}}{\Phi_{1}}\right)\right), \\
& b_{40}=\frac{K^{2}}{105 \pi^{3 / 2}} \exp \left(-t_{0}\left(\frac{1}{\tau}+\frac{1}{\Phi_{1}}\right)\right) .
\end{aligned}
$$

\section{Appendix C}

Coefficients $b_{l m}$ used in Eq. (A.5) when the dipole $\boldsymbol{\mu}$ is included in the plane orthogonal to the $z$ axis:

$$
\begin{aligned}
b_{00}= & \frac{K^{2}}{360 \pi^{3 / 2}} \exp \left(-\frac{t_{0}}{\tau}\right)\left(3 \exp \left(-\frac{t_{0}}{\Phi_{2}}\right)\right. \\
& \left.+\exp \left(-\frac{t_{0}}{\Phi_{1}}\right)+5\right) \\
b_{20}=- & \frac{K^{2} \sqrt{5}}{2520 \pi^{3 / 2}} \exp \left(-\frac{t_{0}}{\tau}\right)\left(6 \exp \left(-\frac{t_{0}}{\Phi_{2}}\right)\right. \\
& \left.+5 \exp \left(-\frac{t_{0}}{\Phi_{1}}\right)+7\right), \\
b_{2 \pm 2}= \pm & \frac{K^{2} \sqrt{30}}{5040 \pi^{3 / 2}} \exp \left(-\frac{t_{0}}{\tau}\right)\left(9 \exp \left(-\frac{t_{0}}{\Phi_{2}}\right)\right. \\
& \left.+2 \exp \left(-\frac{t_{0}}{\Phi_{1}}\right)+7\right), \\
b_{40}= & \frac{K^{2}}{840 \pi^{3 / 2}} \exp \left(-\frac{t_{0}}{\tau}\right)\left(\exp \left(-\frac{t_{0}}{\Phi_{2}}\right)\right. \\
& \left.+2 \exp \left(-\frac{t_{0}}{\Phi_{1}}\right)\right), \\
b_{4 \pm 2}= & \frac{K^{2}}{360 \pi^{3 / 2}} \exp \left(-\frac{t_{0}}{\tau}\right)\left(3 \exp \left(-\frac{t_{0}}{\Phi_{2}}\right)\right. \\
& \left.+\exp \left(-\frac{t_{0}}{\Phi_{1}}\right)+5\right), \\
b_{4 \pm 4}= & \frac{K^{2} \sqrt{70}}{1680 \pi^{3 / 2}} \exp \left(-\frac{t_{0}}{\tau}\right) \exp \left(-\frac{t_{0}}{\Phi_{2}}\right) .
\end{aligned}
$$

\section{References}

[1] J. Lakowicz, Principles of Fluorescence Spectroscopy, Plenum Press, New York, 1985.

[2] J. Simon, Ultrafast Dynamics of Chemical Systems, Kluwer, Dordrecht, 1994.

[3] G.R. Fleming, Chemical Applications of Ultrafast Spectroscopy, Oxford, New York, 1986.

[4] C.V. Shank, E. Ippen, Appl. Phys. Lett. 26 (1975) 62.

[5] D. Waldeck, A.J. Cross, D.B. Mc Donald, G.R. Fleming, J. Chem. Phys. 74 (1981) 3381.

[6] D. Waldeck, G.R. Fleming, J. Phys. Chem. 85 (1981) 2614.

[7] A.J. Cross, D.H. Waldeck, G.R. Fleming, J. Chem. Phys. 78 (11) (1983) 6455.

[8] R.S. Hartman, W.M. Konitsky, D.H. Waldeck, Y.J. Chang, E.W. Castner Jr., J. Chem. Phys. 106 (1997) 7920.

[9] H.E. Lessing, A. von Jena, Laser Handbook, M.L. Stitch, vol. 3, North Holland, New York, 1979.

[10] R.S. Moog, D.L. Bankert, M. Maroncelli, J. Phys. Chem. 97 (1993) 1496.

[11] M.L. Horng, J.A. Gardecki, M. Maroncelli, J. Phys. Chem. A 101 (1997) 1030.

[12] A. Kawski, Crit. Rev. Anal. Chem. 23 (6) (1993) 459.

[13] J. Torga, J. Etcheverry, M. Marconi, Optics Comm. 143 (1997) 234.

[14] J. Torga, M. Marconi, Proc. International Conference on Laser '96, 1996.

[15] J. Torga, J. Etcheverry, M. Marconi, Proc. International Conference on Laser '97, 1997.

[16] T. Karstens, K. Kobs, J. Phys. Chem. 84 (1980) 1871.

[17] M. Ehrenberg, R. Rigler, Chem. Phys. Lett. 14 (5) (1972) 539.

[18] M.E. Rose, Elementary Theory of Angular Momentum, Wiley, New York, 1957.

[19] T.J. Chuang, K. Eisenthal, J. Chem. Phys. 57 (1972) 5094.

[20] L. Favro, Phys. Rev. 119 (1960) 53.

[21] T. Tao, Biopolymers 8 (1969) 609. 